\title{
Update on Bone Grafting Materials Used in Dentistry in the Bone Healing Process: Our Experience from Translational Studies to Their Clinical Use
}

\author{
Gretel G. Pellegrini, Andrea S. Mattiuzzi, \\ Miguel A. Pellegrini, Luis A. Corso, \\ Cintya P. Contreras Morales, \\ Elizabeth Arandia Osinaga and Susana N. Zeni
}

Additional information is available at the end of the chapter

http://dx.doi.org/10.5772/intechopen.79261

\begin{abstract}
The use of bone grafts is important to preserve the alveolar bone ridge height and volume indispensable for dental implant placement. Despite the highly successful outcomes for the implant-supported overdentures, it seems that a majority of edentulous individuals have not pursued implant-based rehabilitation. Among the reasons cited for this, discrepancy between highly successful therapy and its acceptance is the cost of the treatment. Therefore, the development of biomaterials for bone grafting with comparable characteristics and biological effects than those renowned internationally is necessary. In addition, domestic manufacture would reduce the high costs in public health arising from the application of these biomaterials in the dental field. The aim of the following chapter is to offer an update on one bone grafting material frequently used in dentistry through an assessment of anorganic bovine bone graft in small and medium experimental models as well as its clinical use.
\end{abstract}

Keywords: bovine bone graft, new bone formation, critical size bone defect, sinus augmentation, osteoconduction

\section{Introduction}

Bone graft is an implanted material that promotes bone healing alone or in combination with other material(s) [1]. The use and success of bone grafts in the medical field date from the 
beginning of the nineteenth century. In this regard, Albee in 1915 had depicted that there were successfully bone transplants in animal models, as well as in humans, since 1809 [2]. Nowadays, bone graft implantation is the main treatment modality for bone defect repair and reconstruction [3]. In oral and maxillofacial areas, bone grafting aims to replace the volumetric bone loss that frequently occurs by systemic pathologies, periodontal defects, and tooth loss [4].

The mechanisms underlying the bone healing promoted by a bone graft are osteogenesis (osteodifferentiation and subsequent new bone formation by donor cells derived from the host or graft), osteoinduction (induction of undifferentiated and pluripotent cells to develop osteogenesis into the bone-forming cell lineage), and osteoconduction (the ability to support the attachment of osteoblast and osteoprogenitor cells and the migration and ingrowth of these cells within the three dimensional architecture of the graft) [5, 6], in combination or alone [7].

Bone grafting materials are classified as autografts (derived from the same individual), allografts (derived from a different individual from the same species), xenografts (derived from a different species), and alloplasts (derived from synthetic sources) [8]. Autografts are the "gold standard" in the reconstruction of bone defects due to their osteoconductive as well as osteoinductive properties [9]. Although they present excellent biological outcomes, they also have a number of drawbacks. In this regard, the use of autografts increases the operative time due to graft harvest, increases the donor site morbidity, and increases the graft resorption. In addition, they represent a big challenge for the operator, since they need to be mold and have limited availability, especially in the pediatric population [10]. Allografts are typically obtained from human cadavers and require to be processed before being used [11, 12]. Allograft bone is available as cortical, cancellous, corticocancellous forms, or as demineralized bone matrix. They can be processed as mineralized or demineralized, fresh, fresh-frozen, or freeze-dried forms $[13,14]$. Among the benefits of allografts are their availability in different shapes and sizes. This is particularly advantageous, since it avoids donor site morbidity [15]. The major disadvantages of allografts are related to the transmission of diseases and the graft rejection. In order to decrease the risk of transmitting infectious diseases, allografts need to be treated. The techniques employed include treatment with hypotonic solutions, acetone, ethylene oxide, or gamma irradiation that may eliminate cellular and viral particles [16]. However, these processes eliminate the bone cells and denature proteins present in the graft altering the osteoconductive and osteoinductive properties and eliminating the osteogenic properties [17]. In addition, allografts are capable to induce immunological reactions that interfere with the bone healing process leading to rejection of the graft [15, 18-20]. Xenografts are frequently derived from bovine, porcine, and coral sources [7]. The effectiveness of different bone processing techniques has made possible the use of these materials for medical applications [21, 22]. Bovine bone is one of the most popularly used xenografts. This source material is desirable because it is readily available an inexpensive. However, bovine bone grafts require proper preparation to avoid risks such as transmission of zoonoses [23]. Several studies have shown that organic or inorganic matrix derived from bovine bone is biocompatible and osteoconductive [23, 24]. These important biological properties allow the apposition of newly formed bone by osteoprogenitor cells and the partial remodeling by osteoclasts and osteoblasts of the host [25]. Moreover, the large interconnecting pore volume and its composition encourage the formation and ingrowth of new bone at the implantation sites. Ideally, a synthetic bone graft should be biocompatible and causes minimal fibrotic changes [26]. Synthetic bone grafts 
are osteoconductive and have been shown to integrate to bone [27]. There are many available kinds of synthetic graft materials, including bioactive glasses, a- and b-tricalcium phosphate (TCP), and synthetic hydroxyapatite [27].

Bioactive glass or "bioglasses" has been widely used as bone substitutes because of their ability to join and integrate with the bone tissue by forming a layer of active apatite on its surface, with characteristics similar to bone [28]. These biomaterials are resorbable, and dissolution of their products (soluble silicon and calcium) upregulates seven families of genes present in osteoblasts (bone forming cells), thus promoting osteogenesis [28, 29]. Among synthetic materials, synthetic hydroxyapatite, a crystalline phase of calcium phosphate found naturally in bone minerals, exhibits initial mechanical rigidity and structure and demonstrates osteoconductive, as well as angiogenic properties in vivo [30]. Because of its physicochemical characteristics, synthetic hydroxyapatite is a biocompatible and osteoconductive material [31]. This material allows to keep the space filled extremely well providing a physical matrix for the deposition of new bone. For these reasons, synthetic hydroxyapatite has high success in the fields of biology, medicine, and dentistry.

Due to the high popularity of dental implant surgery, the demand for alveolar ridge reconstruction, including sinus augmentation and immediate implant procedures, increased. This new trend in dentistry for implants boosted the development of new grafting materials in dentistry. Ideally, a bone graft should be biocompatible, biodegradable, osteoconductive, and osteoinductive, structurally similar to bone, easy to use, and cost-effective [7]. Within these parameters, a growing number of bone graft alternatives are commercially available and frequently used in dentistry.

Different types of bone grafts are available in the international market. However, it is essential to have a wide variety of them to improve the competitivity of each product in terms of quality, commercial value, and clinical use. Therefore, the development of biomaterials for bone grafting, produced by domestic manufactures, with comparable characteristics and biological effects than those renowned internationally, is necessary in order to reduce the high costs in public health arising from the application of these biomaterials in the dental field. The aim of the following chapter is to offer an update on one bone grafting material frequently used in dentistry through an assessment of an organic bovine bone graft in small and medium experimental models, as well as its clinical use.

\section{Translational studies}

We evaluated and compared the effects of a bovine bone graft made in Argentina with a commercial bovine bone graft recognized for its osteoconductive effects, on bone healing process in experimental models in rats (experiment 1) and rabbits (experiment 2).

\subsection{Materials and methods}

The research and all procedures involving live animals were processed after approval by the Clinical Hospital, School of Medicine, University of Buenos Aires, Argentina. Animals were 
maintained in keeping with the National Institutes of Health Guide for the Care and Use of Laboratory Animals.

\subsubsection{Experiment 1 in rats}

A total of 12 young male adult Wistar rats (175 $\pm 10 \mathrm{~g})$ were housed at room temperature $\left(21 \pm 1^{\circ} \mathrm{C}\right), 55 \pm 10 \%$ humidity, under 12 -hour light/dark cycles. They were fed a standard rodent diet (Ganave SA, Argentina) and deionized water "ad libitum." Body weight was recorded 3 times per week.

\subsubsection{Surgical procedure}

Rats were anesthetized by intraperitoneal injection with ketamine hydrochloride $[0.1 \mathrm{mg} / 100 \mathrm{~g}$ body weight (BW)] and acepromazine maleate $(0.1 \mathrm{mg} / 100 \mathrm{~g} \mathrm{BW})$, (Holliday-Scott S A, Buenos Aires, Argentina). Hind legs were shaved, and the medial aspect of tibiae was exposed. A critical size bone defect (CSBD) $(1.6 \mathrm{~mm} \times 2 \mathrm{~mm})$ was made with a fissure bur under copious irrigation with saline [32]. Bone defects were filled as follows: Group $1(n=6)$ : The right tibia of each rat was filled with the bovine bone graft Synergy Bone Matrix (SBM), (Synergy Bone Matrix, Odontit Implant Systems, Argentina, Lot No: E11121216), while the left tibia was unfilled and used as control. Group $2(n=6)$ : The right tibia of each rat was filled with the bovine bone substitute SBM (Lot No: E11121216) and the left tibia with the bovine bone substitute Bio-Oss (BO), (Bio-Oss, Geistlich, Switzerland, Lot No 100238).

Taking into account that the remodeling phase in the rat takes approximately 21 days, a healing period of 4 weeks was used to assess the late healing response. Animals were sacrificed after 24 days by carbon dioxide inhalation.

\subsubsection{Experiment 2 in rabbits}

A total of 15 adult male New Zealand rabbits (weight $3.0 \pm 0.5 \mathrm{~kg}$ ) were housed in individual cages with grid floating floor of stainless steel in a $12 \mathrm{~m}^{2}$ insulated and equipped room with a continuous extraction system air renewal. They were maintained at room temperature $\left(21 \pm 1^{\circ} \mathrm{C}\right), 55 \pm 10 \%$ relative humidity, and under 12 -hours light/dark cycles. Cleaning and disinfection of excreta trays are made daily. Prior to surgery, animals had an adaptation period of 10 days. Pelleted commercial diet and deionized water were supplied ad libitum throughout the experiment with the exception of the first 3 days after surgery, in which they were fed with fresh leafy vegetables. Body weight was assessed 3 times per week during the morning.

\subsubsection{Surgical procedure}

All surgical procedures were carried out under aseptic conditions and in accordance with ISO 10993-6: 2007. The experimental surgery was performed under general anesthesia by an intramuscular injection of $1 \mathrm{mg} / \mathrm{kg}$ of acepromazine maleate and an intramuscular injection of ketamine hydrochloride and xylazine (Holliday-Scott S A, Buenos Aires, Argentina), at a dose of 35 and $5 \mathrm{mg} / \mathrm{kg}$, respectively. The surgical site was shaved and scrubbed with iodine. A parallel skin incision was made along the inferior border of the mandible on both sides. 
After exposing the masseter muscle, subperiosteal elevation of the muscles detachment was performed exposing the body of the mandible. A standardized CSBD of $5 \mathrm{~mm}$ diameter on each side of the mandible was created with a slowly rotating trephine bur, under constant and copious irrigation with saline [33,34]. Once the bone was excised, each biomaterial (SBM, Lot No: H13010002 or BO, Lot No 100148) was implanted in the corresponding side of the mandible according to the following groups: Control group: CSBD without bone graft; SBM group: CSBD in the right mandible filled with SBM; BO group: CSBD in the right mandible filed with BO. Animals were sacrificed at 4, 8, and 12 weeks postsurgery by a lethal intravenous administration of sodium pentobarbital (Euthanyle ${ }^{\circledR}$ Brouwer, S.A., Argentina).

Postoperatively, rabbits were given a $0.5 \mathrm{ml}$ subcutaneous injection of penicillin and streptomycin antibiotic (Dipenisol Retard, Bayer Argentina) every 48 hours for 7 days. In order to supplement the analgesia, intramuscular injection of $1 \mathrm{mg} / \mathrm{kg}$ of Nalbufina (Nalbufine 10 Richmond S.A, Argentina) was administered as premedication and every 12 hours during 3 days postsurgery.

The 5-mm diameter CSBD created in the rabbit jaw for implantation with bone grafts is generally considered to be the appropriate critical size to evaluate bone graft materials [35-37]. Bone remodeling in the rabbit is approximately three times faster than in humans. Therefore, a healing period of 4 weeks was considered appropriate to evaluate the early response to bone healing. Likewise, for the evaluation of delayed bone repair, in relation to the amount of neoformed bone and the reabsorption of the biomaterials used, the period of 8 to 12 weeks was considered [38, 39].

In both experiments, the wound was closed with absorbable sutures, after filling each bone defect. Each animal surgical area was disinfected with iodine, and animal behavior was observed and recorded.

\subsubsection{Bone mineral density evaluation in rats}

Total skeleton bone mineral density and bone mineral content (BMD and BMC, respectively) were measured "in vivo" under light anesthesia at the end of the experiment (day 24) using a total body scanner with software designed specifically for small animals (DPX Alpha 8034, Small Animal Softer, Lunar Radiation Corp, Madison, WI) following a previously described technique [40]. Rats were scanned under light anesthesia. The precision of the software in determining total body BMD was assessed by measuring one rat five times after repositioning between scans, both on the same and on different days [40]. The coefficient of variation (CV) was $0.9 \%$ for total skeleton BMD. A specific region of interest (ROI) was manually traced at the site of the critical size bone defect for the first animal evaluated. Once established the ROI for the first animal, we used the same ROI to evaluate the BMD at the site of the bone defect in all the animals. The BMD CV of the studied area was 3.5\% for the proximal tibia. All the analyses were carried out by the same technician in order to minimize inter-observer variation.

\subsubsection{Histological and histomorphometrical analysis}

At the end of the experimental period, tibiae (experiment 1) and mandibles (experiment 2) were removed and cleaned of soft tissue. All specimens were prepared for histological evaluation according to a standard protocol for undecalcified sections, as previously described [41], and stained with hematoxylin-eosin. The analysis was "blinded" performed with respect to 
the rendered treatment. Images of the histological sections were captured by a digital camera (Olympus DP 10; Olympus Optical, Tokyo, Japan) connected to a light microscope (Olympus CX 31; Olympus Optical). Digital images were saved for static histomorphometrical analysis (experiment 2) using Image-Pro Plus 4.5 software. The following criteria were used to standardize the analysis: the total area (TA) to be analyzed was delineated on the composite image. The TA $\left(\mathrm{mm}^{2}\right)$ corresponded to the area of the mandible where the surgical defect was previously created, and it was considered $100 \%$ of the area to be analyzed. The newly formed bone area (NFBA) and the remaining graft particles area (RGPA) were then delineated. Both, NFBA and RGPA, were located entirely within the confines of the TA. The NFBA and RPGA were also calculated in $\mathrm{mm}^{2}$ and the percentage calculated according to the following formula: 100-NFBA/TA. Values obtained from each animal were used to calculate the means and SD of each control and experimental group. We evaluated bone volume (\% BV/TV): the percentage of cancellous bone within the total measured area and the percentage of remaining particles (\% RP/TV) of either SBM or BO.

\subsubsection{Biomechanical tests in rabbits}

Biomechanical measurements were performed using a three-point bending test (Instron 4411, Universal Testing Materials). The equipment consists of a load frame in which is placed the material to be test (test tube) and a control console that provides calibration controls, programming, and test operation. The installed load cell allows the measurement of compressive forces exerted on the crosshead specimen. IX Series software was used to pick up data analysis of testing bone material. Control, SBM, and BO specimens were cleaned of soft tissues and cut in squares of $20 \times 20 \mathrm{~mm}$ in the area where the bone defect was done. Bones were weighed and measured. Then they were placed one by one on the rollers, and the bone fracture was performed to evaluate elastic modulus (Mpa) and shear modulus (Mpa). Compression test was performed to evaluate compressive strength $\left(\mathrm{KgF} / \mathrm{mm}^{2}\right)$.

\subsubsection{Statistical analysis}

Results were expressed as mean \pm standard deviation (SE). Data were analyzed using parametric tests according to data distribution and "a posteriori" tests. Data were analyzed using one-way analysis of variance (ANOVA). The Bonferroni multiple comparisons test was performed when significant differences were found. Student's t-test for independent samples was used to compare both bone grafts at each end point. Statistical analyses were performed using SPSS version 19 (SPSS Inc., Chicago, IL, USA). $\mathrm{p}<0.05$ was considered significant.

\section{Results}

\subsection{Experiment 1 in rats}

\subsubsection{Bone mineral density evaluation}

Non statistically significant differences were found in BMC and BMD of tibiae filled with SBM and BO (Figure 1), whereas SBM and BO exhibited higher BMC and BMD than the control group (Figure 1). 
A

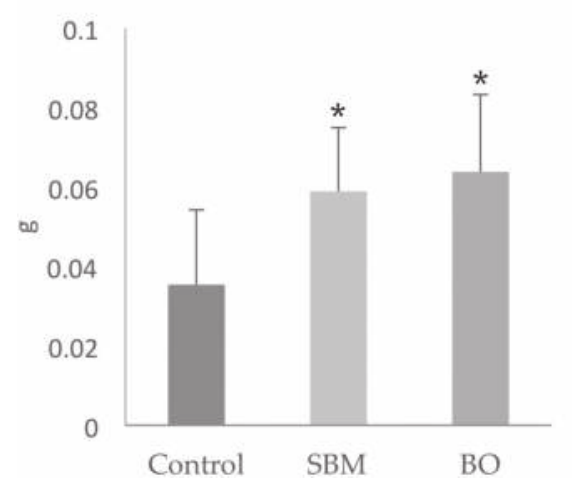

B

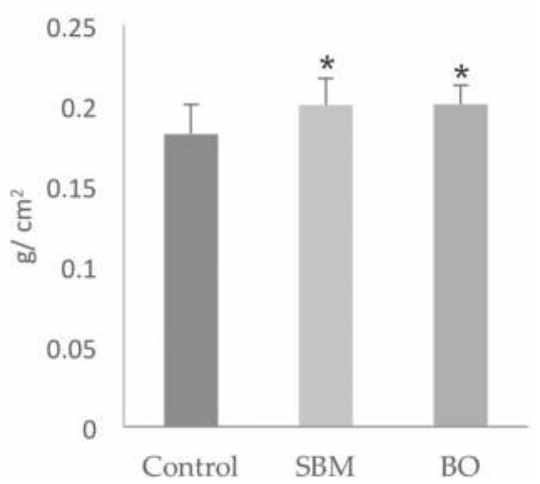

Figure 1. Total skeleton bone mineral content (A) and bone mineral density (B) in control, Bio-Oss (BO) and synergy bone matrix groups (SBM) $\left({ }^{*} \mathrm{p}<0.05\right.$ vs. control).

\subsubsection{Histological analysis}

Cross-sections of tibiae showed remaining particles of each bovine bone graft in the area of the CSBD. Multiple particles of either SBM or BO, of different shapes and sizes, surrounded by laminar bone tissue were observed in the bone medullary space (Figure 2A, B and C). This finding indicates that both bone substitutes were osteoconductive. Proper bone healing was observed in tibiae from both groups. No signs of inflammation were observed; this result suggests biocompatibility (Figure 2A, B and C). After 4 weeks, blood vessels with small angiogenesis and revascularization foci formed in the CSBD implanted with either SBM or BO. The samples also showed mature Haversian systems forming a thin interface within the NBF represented by bone growth and surfaces covered by osteoblasts and fibroblast-like cells surrounding the bone grafts, which implied active osteogenesis. The present study suggests that the bovine bone graft SBM presented similar properties of biocompatibility without inflammatory signs to that of BO. Moreover, SBM also exhibited similar osteoconductive properties to $\mathrm{BO}$, allowing a normal bone formation surrounding the particles.

\subsection{Experiment 2 in rabbits}

\subsubsection{Histological analysis}

As expected, control rabbits did not exhibited NBF at any of the studied times. Instead histological samples exhibited normal development of fatty bone marrow with occasional remnants of hematopoiesis foci in the site of the CSBD (Figure 3A, D and G). CZBD filled with each of the bovine bone grafts did not evidenced "foreign body" reaction at any of the studied times. Bone healing over time was accompanied by a progressive inflammatory response consistent with the expected histological stages of repairing. In addition, SBM and BO groups presented NBF characterized by trabecular bone growth and the presence of osteoblasts and fibroblastlike cells (Figure 3). Both samples exhibited blood vessel formation with small angiogenesis and revascularization foci and Haversian mature systems the implanted grafts, forming a 


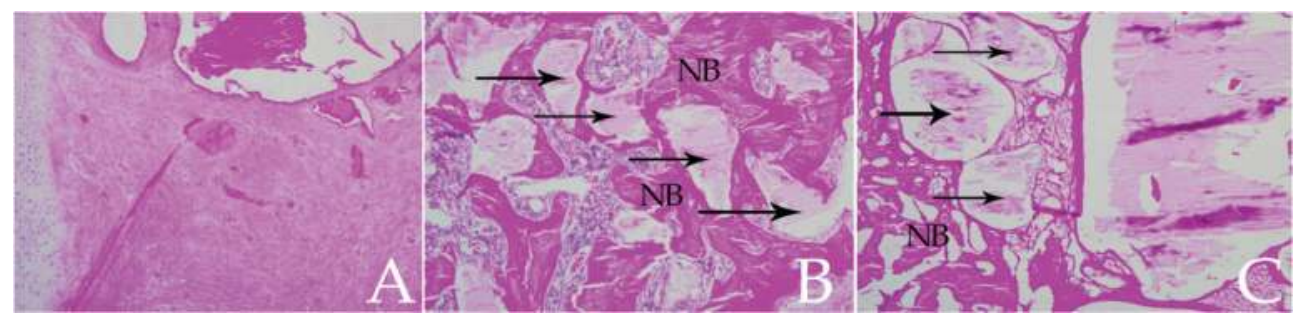

Figure 2. Histological evaluation in rats. Photomicrography of the critical-sized bone defects at $10 \times$ or $40 \times$ magnification and stained with Hematoxylin-Eosin. A: Control group exhibited the presence of fibrous tissue. B: Critical-sized bone defect filled with synergy bone matrix (SBM). C: Critical-sized bone defect filled with Bio-Oss (BO). Black arrows indicate SBM or BO particles; NB: new bone formation surrounding the particles.

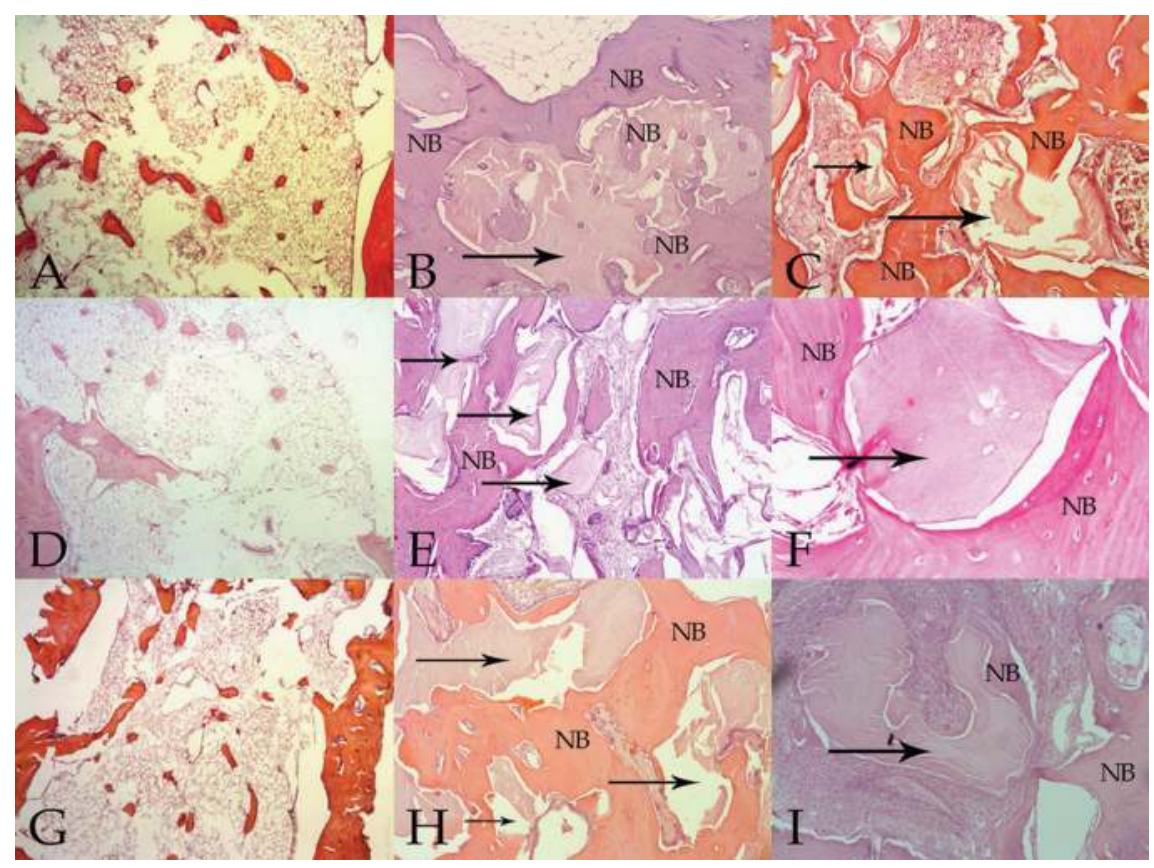

Figure 3. Histological evaluation in rabbits. Photomicrography of the critical-sized bone defects (CSBD) at $4 \times$, 10× or 40× magnification and stained with Hematoxylin-Eosin. Control group exhibited the presence of fibrous tissue at 4 (A), 8 (D) and $12(\mathrm{G})$ weeks. CSBD filled with synergy bone matrix (SBM) and Bio-Oss (BO) showed new bone formation surrounding each particle at 4,8 and 12 weeks. B, E and H: SBM at 4, 8 and 12 weeks. C, F and I: BO at 4, 8 and 12 weeks. Black arrows indicate SBM or BO particles; NB: new bone formation surrounding the particles.

thin interface with the bone forming tissue. The presence of traces of bone substitutes and the formation of blood vessels with small foci of angiogenesis and revascularization was also evident. Moreover, we observed the presence of mature Haversian systems around the bone grafts (Figure 3). These findings indicate that SBM and BO are osteoconductive and histologically substantially equivalent. 


\subsubsection{Histomorphometrical analysis}

Histomorphometrical analysis showed an increase in new bone formation (\% BV/TV) that was time dependent for both bone grafts compared to the control group $(\mathrm{p}<0.05)$ (Figure 4A). A time dependent reduction in the percentage of remnant particles of each device (\% RP/TV) was also observed. As control group was not filled with any of the grafts, RP/TV remained in 0 . Since the size of the granules of SBM was bigger than that of $\mathrm{BO}$, it is speculated that this factor contributed to the low percentage of RP/TV observed for $\mathrm{BO}$ (Figure 4B).

\subsubsection{Biomechanical tests}

Both grafting materials exhibited a significant increase in elastic modulus, shear modulus, and compressive strength at 4, 8, and 12 weeks (Figure 5A, B, and C). This finding suggests that the biomechanical properties of the newly formed bone were equivalent for the two grafts evaluated. Moreover, the quality of the newly formed bone was superior to that presented by the control group.

A

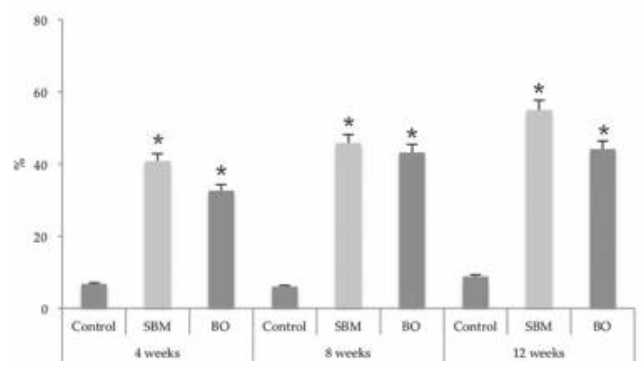

B

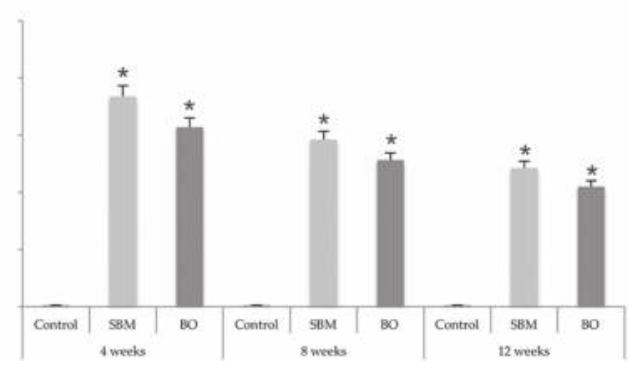

Figure 4. New bone formation (A). Histomorphometrical analysis of rabbit mandible showed an increase in bone formation in the critical sized bone defects filled with synergy bone matrix (SBM) or Bio-Oss (BO), ${ }^{*} \mathrm{p}<0.05$ by one-way ANOVA, followed by Bonferroni multiple comparisons test. Remnant particles of each device (B). Histomorphometrical analysis of rabbit mandible showed a time-dependent decrease in the remaining particles in the critical sized bone defects filled with SBM and BO, $\mathrm{p}=$ N.S between SBM and BO by one-way ANOVA.

A

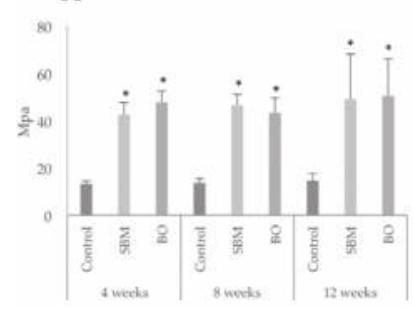

B

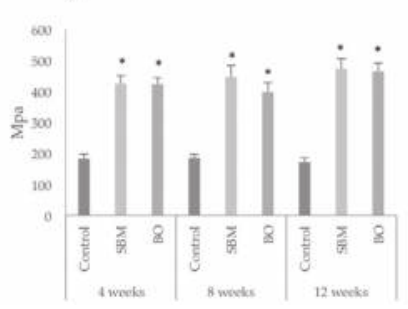

C

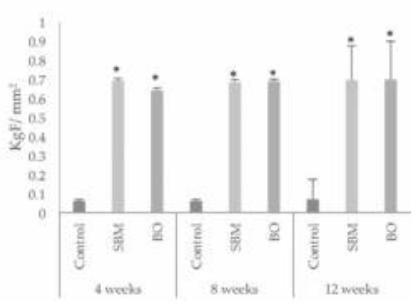

Figure 5. Biomechanical tests. The new bone formed in the critical sized bone defects filled with synergy bone matrix (SBM) and Bio-Oss (BO) presented better quality than the control group exhibited by higher elastic modulus (A), shear modulus (B) and compressive strength (C). ${ }^{*} \mathrm{p}<0.05$ by one-way ANOVA, followed by Bonferroni multiple comparisons test. 


\section{Case report}

A 54-year-old female patient was referred to the Department of Clinical Operative and Prosthesis II, Dental School, University of Buenos Aires, Buenos Aires, Argentina, for the rehabilitation of the edentulous maxilla. Radiographic and cone beam computed tomography (CBCT) exhibited severe atrophy in the posterior region of the maxilla (Figure 6). The medical history did not reveal any systemic disease, and the patient did not reported to be under any medication. The patient aimed to rehabilitate the upper jaw with a fixed implant-supported

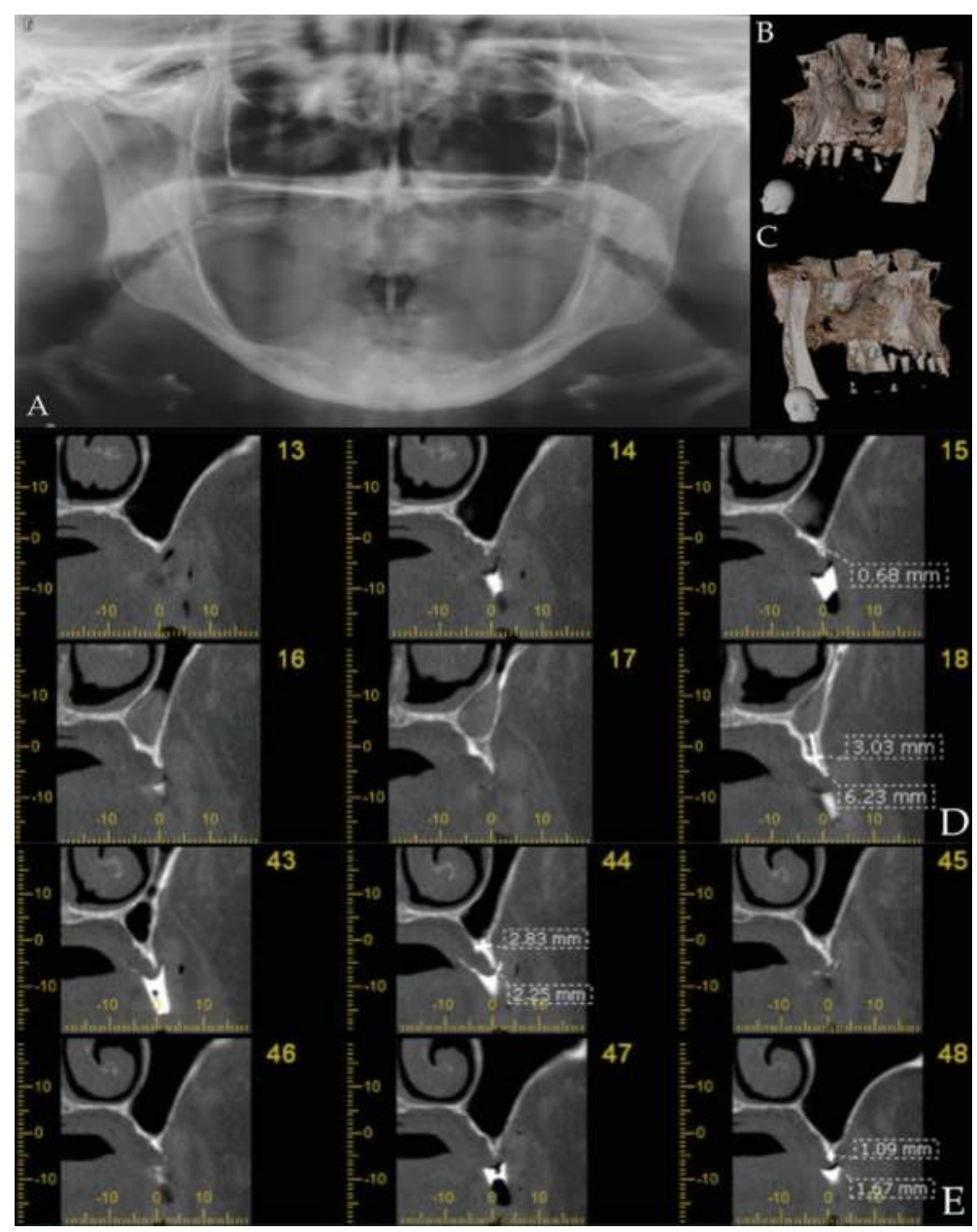

Figure 6. Preoperative diagnostic images. All images exhibited a dramatic loss of bone in the upper left and right maxilla. A: Panoramic X-ray showing edentulous maxilla and mandible. B, C. 3-D reconstruction of the left (B) and right (C) maxilla with the surgical stent. D, E: coronal cut from a cone beam computed tomography scan from the left (D) and right (E) maxilla. 
prosthesis. The proposed treatment plan was divided in two stages. The first stage included the confection of a complete upper denture, as well as a surgical and radiological stent, and the reconstruction of the posterior maxillary alveolar ridge. The second stage, after 6 months, consisted in the placement of four dental implants in the posterior maxilla. All the clinical procedures were conducted under the patient's written informed consent. The purpose of this clinical case report was to provide clinical evidence of the efficacy of a new bovine bone graft in the bone healing process.

\subsection{Sinus elevation surgery and guided tissue regeneration}

The bilateral sinus elevation procedure was performed using the technique previously described by Tatum [42]. Briefly, after anesthetized with infiltrative local carticaine hydrochloride $4 \%$ with adrenaline 1:100.000 (Totalcaína Forte, Microsules Bernabó, Argentina), a mucoperiosteal flap was elevated with releasing vertical incisions. Once exposed the buccal wall of the remaining alveolar process and the anterolateral wall of the Highmore antrum, a surgical stent was used to locate the lateral window. An oval osteotomy was performed with high speed handpiece and a round diamond bur under copious irrigation with saline, leaving a "bone island," in the lateral wall of the sinus, attached to the Schneider membrane (Figure 7). This fragment of bone was then turned medially and positioned toward the sinus floor. The sinus membrane was then elevated across the floor and up the medial wall. A bilateral guided bone regeneration procedure was performed using a mixture of SBM. The graft was covered with a rebsorbable collagen membrane (BioCollagen, Bioteck, Italy). Finally, the flap was repositioned and sutured without tension. The patient was instructed to perform oral hygiene and to rinse 2 times a day during 7 days with chlorhexidine digluconate $0.12 \%$ for disinfection of the surgical wound. Amoxicillin-clavulanate $875 \mathrm{mg}$ was prescribed twice a day for 7 days, and $500 \mathrm{mg}$ of naproxen was administered every 8-12 hours for 5 days to control postoperative pain. Soft diet was also recommended. The sutures were removed after 7 days. CBCT scans and panoramic x-rays were obtained preoperative, 6 months after stage 1 , and 4 months after stage 2 . A biopsy of each treated area was taken with a trephine bur during the implant placement surgery.

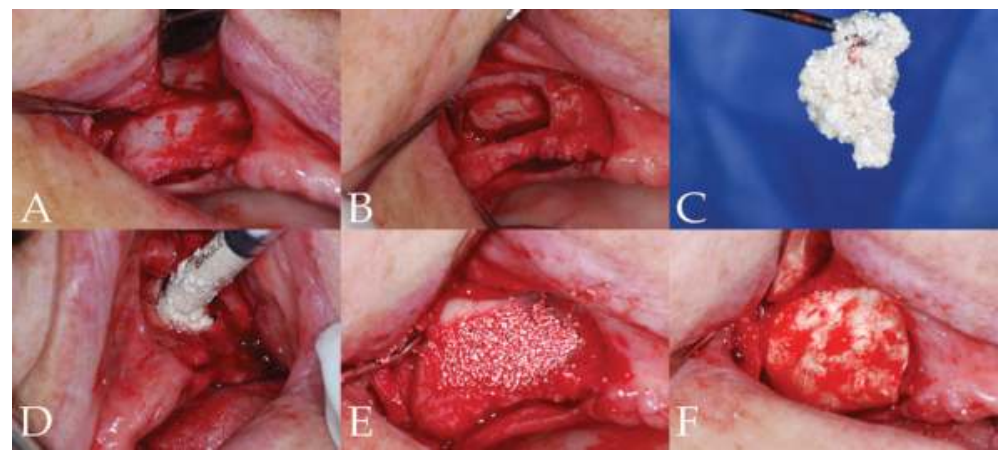

Figure 7. Sinus elevation surgery and guided tissue regeneration. A: Elevation of a mucoperiosteal flap. B: Oval osteotomy and "bone island" in the lateral wall of the sinus attached to the Schneider membrane. C: Synergy bone matrix (SBM). D, E: Placement of SBM for guided bone regeneration. F. The graft was covered with a rebsorbable collagen membrane. 


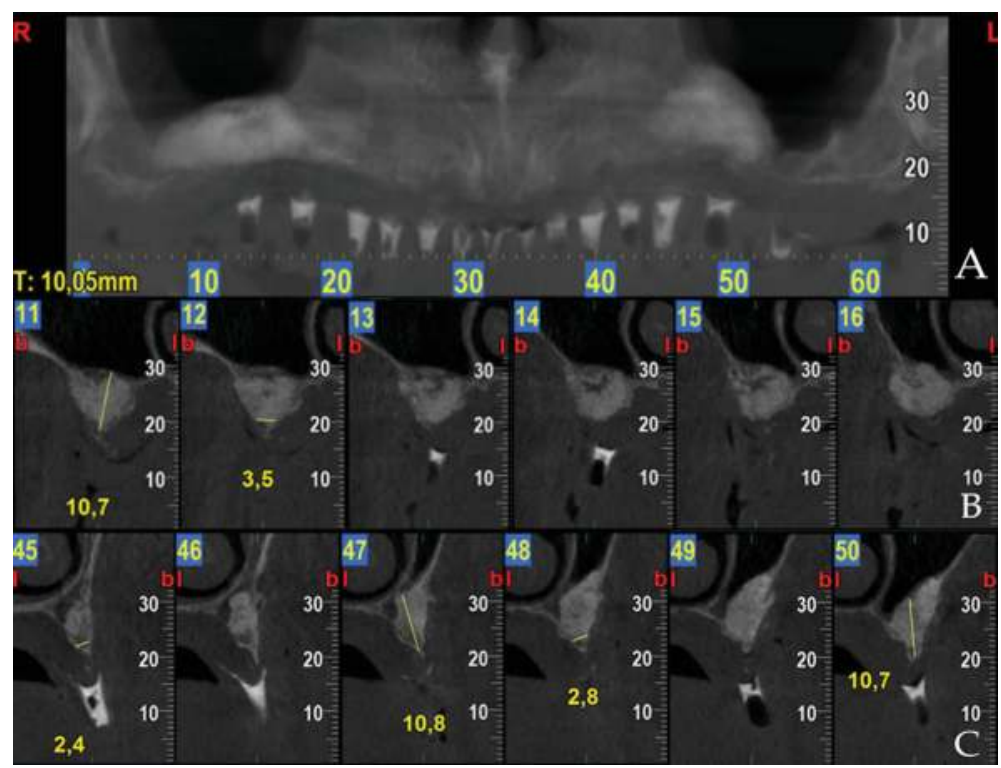

Figure 8. Postoperative CBCT (6 months after the sinus elevation surgery). A, B, C: There was an increase of $10.7 \mathrm{~mm}$ and $10.8 \mathrm{~mm}$ in the height of the alveolar crest, and an increase in the alveolar crest width of $3.5 \mathrm{~mm}$ and $2.8 \mathrm{~mm}$ in the right and left side, respectively.

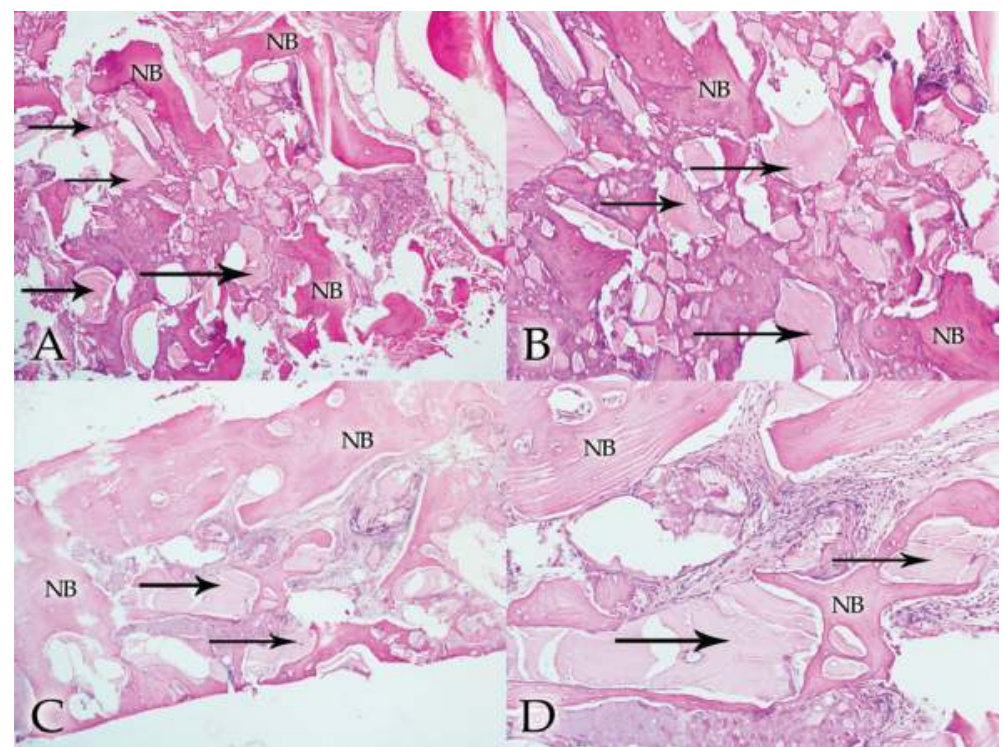

Figure 9. Histological evaluation of the areas grafted with synergy bone matrix (SBM) at $4 \times, 10 \times$ or $20 \times$ magnification and stained with Hematoxylin-Eosin. New bone formation surrounding each particle was observed in right (A, B) and left (C, D) grafted sinus. Black arrows indicate SBM particles. NB: new bone formation. 
During the first surgical stage, a postoperative follow-up 7 days after the procedure revealed that the edges of the flap wounds faced each other, and there were no signs of dehiscence or inflammation. The patient did not report any discomfort, pain, or inflammation of the treated areas. The postoperative $\mathrm{CBCT}$, taken 6 months after this surgery, exhibited an increase of $10.7 \mathrm{~mm}$ and $10.8 \mathrm{~mm}$ in the height of the alveolar crest and an increase in the alveolar crest width of $3.5 \mathrm{~mm}$ and $2.8 \mathrm{~mm}$ in the right and left side, respectively (Figure 8). Six months after the sinus lift surgery, dental implants were placed in the areas that received the bone graft (stage 2). Dental implants in the areas grafted achieved primary stability, indicating that there was an accurate bone quality after the placement of the bone graft. Consistent with the digital imaging findings, histological evaluation of the bone samples retrieved during the implant surgery revealed that SBM particles were osteoconductive. All particles were surrounded by new bone formation (Figure 9). There were fibro-angiogenic and fibrous areas associated to

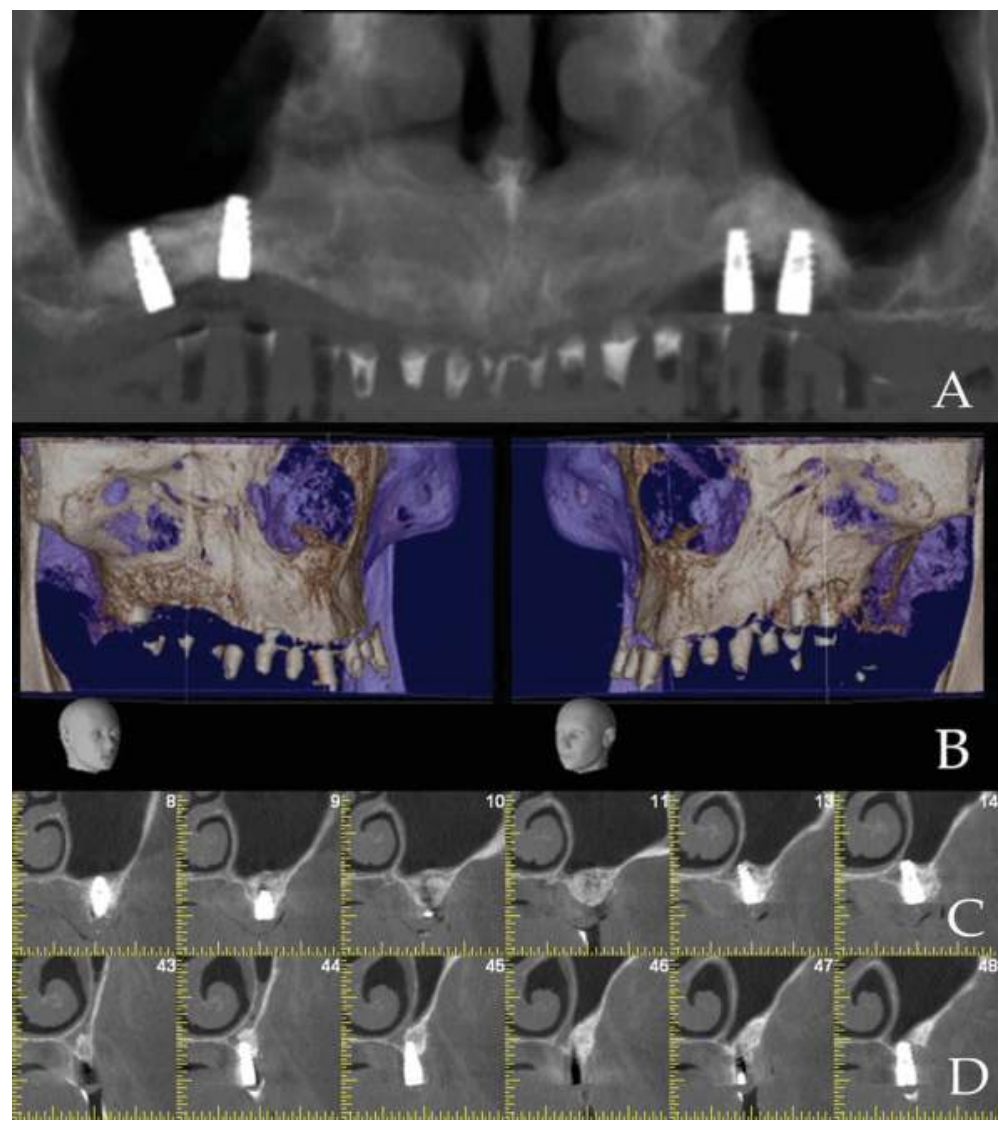

Figure 10. Post-dental implant placement diagnostic images. All images showed bone gain in both sides of the maxilla that persisted after the placement of dental implants. A: Panoramic X-ray from a CBCT showing the increase in alveolar bone height and dental implants on the right and left side. B: Reconstruction of the left and right maxilla with the surgical stent. C, D: Coronal cut from a CBCT scan from the left (C) and right (D) maxilla. 
SBM, as well as gradual regression of associated fibrosis. The bone formation pattern was lamellar and trabecular, and the presence of osteoblast at the surface of the trabeculae, as well as osteocytes, was also observed. There were no signs of inflammation or bone sequestrae.

Postoperative 4-month control digital images exhibited osseointegration of the implants (Figure 10). No peri-implant radiolucencies were observed. The regenerated bone gain by the graft placement in both sides was preserved (Figure 10). Clinical assessment of the dental implants did not exhibited mobility of the implants, and a solid-deaf sound when performing percussion tests showed proper bone healing. The patient did not report pain, and there was no leakage of purulent material or signs of inflammation. In addition, the grafted bone presented the similar density than the perisinusal bone at both sides.

\section{Discussion}

The results of the present report provide evidence for the biocompatibility and osteoconductive properties of Synergy Bone Matrix. Bone graft implantation is the main treatment modality for bone defect repair and reconstruction [3]. In this sense, demineralized bovine bone offers excellent biocompatibility and physicochemical properties due to its mineral similarity with the host tissues [43].

SBM and BO are inorganic bovine bone xenografts indicated for bone defects filling due to their osteoconductive properties. In experimental models, the bone defect above a critical size requires a scaffold to guide bone repair. Deproteinized bovine bone mineral is osteoconductive and provides excellent biocompatibility because it has similar physicochemical characteristics to that of the mineral component of the original bone [3]. These two important biological properties allow apposition of new bone formed by osteoprogenitor cells located in the host tissue. It is noteworthy that bovine bone inorganic-phase not only promotes the deposition of calcium and phosphate ions but also is partially remodeled by osteoclasts and osteoblasts of the host [25]. In addition, the large interconnecting pore volume and its composition encourage the formation and ingrowth of new bone at the implantation sites.

$\mathrm{BO}$ is a recognized commercial bone defect filling material with osteoconductive properties. Under our experimental conditions, SBM showed similar osteoconductive properties to BO. In this regard, our histological findings showed neovascularization in the area implanted with either SBM or BO. This finding suggests that the new bone graft provided an optimal microenvironment for bone ingrowth. Typically, bone formation starts by bone-forming cells secreting bone matrix (i.e., collagen) into the defect area, followed by mineralization to envelope the implanted graft material [44]. Our results in rats showed that collagen fibers were replaced by mature bone, filling the CSBD, thus confirming active osteogenesis after 24 days postimplantation of the graft.

Similarly to what we observed in rats, histological analysis in rabbits at 4 weeks postimplantation showed the presence of blood vessels and revascularization in the areas implanted with both bone grafts. This finding suggests an optimal microenvironment for bone growth. 
Bone formation begins with the appearance of osteoblasts in the defect area that secretes bone matrix (i.e. collagen); this period is followed by mineralization in order to wrap the implanted graft material [44].

Bone is a dynamic tissue that undergoes remodeling. Bone remodeling is a coupled process that starts with osteoclastic bone resorption followed by osteoblastic bone formation [45]. In this sense, the osteoclastic resorption of the graft is affected by both, the particle size as well as the composition and porosity of the material.

Initially, once the graft material is place, it suffers osteoclastic bone resorption followed by bone formation by osteoblastic action. The porosity of the particles enhances new bone formation by allowing the migration and proliferation of osteoblast and mesenchymal cells [46]. In addition, the microporosity of the particles is believed to enhance ionic exchange with body fluids [46]. This characteristic allows each particle of SBM to serve as a 3-D scaffold, in which osteoblast and osteoprogenitor cells migrate and form bone. Consistent with this, during the first period (4 weeks), we observed active osteogenesis evidencing by the presence of bone surfaces covered by osteoblasts around the implanted bone grafts and the formation of mature haversian systems. Toward the end of the experience, the collagen fibers were replaced by mature bone that filled the CSBD region. These findings indicate that the process of bone regeneration induced by SBM was similar to that induced by BO.

It is important to emphasize the quantity and quality of bone formed after placing a bone graft. On this regard, the increase in rat tibiae BMD indicates that the amount of bone increased in the CSBD filled with SBM or BO. In addition, biomechanical test performed in rabbit mandible exhibited an increase in all parameters, suggesting an enhanced quality of the newly formed bone.

Chackarti et al. observed, in a histomorphometric and micro-CT analysis, that granules from different sizes (small or large), produced the same pattern of bone formation: the bone surrounding the graft connects and produces a network of "bone bridges" among the graft particles [47]. The two used bone grafts had a similar granulometry: 1 to $2 \mathrm{~mm}$ for BO and 0.84 to $2 \mathrm{~mm}$ for SBM. Although BO and SBM are available in small granules as well as blocks, these were considered too small or too large for the rabbit experimental model used. In the present study, the volume of remaining bone graft particles was similar, and the biomechanical results did not show differences between SBM or BO, suggesting that the quality of the bone formed was similar for both products.

The loss of teeth in the posterior area of the maxilla leads to adverse consequences on masticatory function and occlusal balance. These outcomes negatively results in psychophysical conditions associated with temporomandibular joint and muscle diseases. A frequent problem in oral rehabilitation with implant-supported prostheses in the posterior maxilla is the lack of bone volume associated with alveolar ridge resorption or maxillary sinus pneumatization [48]. The reabsorption of the alveolar bone, adjacent to the floor of the maxillary sinus, may be aggravated by the increase in osteoclastic activity that originates in the periosteum of Schneider's membrane, after tooth loss, due to the absence of osteogenesis normally stimulated by the functional load on the bone. In this sense, the bone volume is limited due to the 
pneumatization of the maxillary sinus on one hand and the loss of height and width of the alveolar process on the other. In order to increase the bone volume, the maxillary sinus floor elevation technique is used. It consists in elevating the membrane of the floor of the maxillary sinus and filling the intermediate space with bone substitutes [42] to promote bone formation [49]. The results of this procedure can be affected by the surgical techniques used: simultaneous placement versus delayed implantation of the implant, use of barrier membranes on the lateral window, graft material selection and surface characteristics, and length and width of the implants. Depending on the type of graft, the particles are partially reabsorbed and replaced by the patient's own bone during the healing time [50].

In agreement with Shirmohammadi et al. and Wallace et al. on sinus augmentation utilizing BO as bone graft $[51,52]$, the case report presented here evidences the efficacy of SBM in the bone healing process, showing osteoconductive properties when used as a grafting material for sinus lift elevation. In this regard, biopsies of the grafted areas showed that SBM particles were surrounded by vital new bone, without evidence of inflammation and bone sequestrae after 6 months of implantation. We neither observed inflammation nor thickening of the repaired Schneiderian membrane.

Additional comparative studies with greater number of patients and histomorphometric analysis are needed to determine whether there is any advantage in the use of SBM as opposed to $\mathrm{BO}$ in the survival of implants placed in grafted sinuses.

\section{Conclusion}

The use of bone grafts is important to preserve the alveolar bone ridge height and volume indispensable for dental implant placement. Despite the highly successful outcomes for the implant-supported overdentures, it seems that a majority of edentulous individuals have not pursued implant-based rehabilitation. Among the reasons cited for this discrepancy between highly successful therapy, and its acceptance is the cost of the treatment [53]. Our experimental findings in animal models, as well as the case report, indicate that the bone regeneration process induced by SBM presented similar characteristics in osteoconduction than BO and suggests the use of this material to increase the bone volume of the alveolar crest. The presence of a biomaterial with similar characteristics to those of internationally recognized commercial brands, but developed by the domestic industry, will be an important tool to reduce the high cost of these interventions.

\section{Acknowledgements}

The authors thank Dr. Macarena Gonzales-Chaves for her technical advice in histomorphometrical studies and Mr. Ricardo Orzuza for his technical advice in animal care. We also would like to thank University of Buenos Aires, School of Dentistry, Department of General and Oral Biochemistry, Buenos Aires, Argentina for conducting the densitometric evaluations, 
and University of Buenos Aires, School of Dentistry, Department of Clinical Operative and Prosthesis II, Buenos Aires, Argentina for their assistance in the use of the facilities.

This research was partially funded by National Council of Scientific and Technical Research (CONICET)-University of Buenos Aires. Institute of Immunology, Genetics and Metabolism (INIGEM). School of Pharmacy and Biochemistry- Clinical Hospital "José de San Martín," Buenos Aires, Argentina, and Odontit Implant Systems, Argentina. Synergy Bone Matrix and Bio-Oss were kindly provided by Odontit Implant Systems, Argentina.

\section{Conflict of interest}

All authors state that they have no conflicts of interest.

\section{Author details}

Gretel G. Pellegrini ${ }^{1,2 *}$, Andrea S. Mattiuzzi ${ }^{3}$, Miguel A. Pellegrini ${ }^{1}$, Luis A. Corso ${ }^{3}$, Cintya P. Contreras Morales ${ }^{3}$, Elizabeth Arandia Osinaga ${ }^{3}$ and Susana N. Zeni ${ }^{1,2}$

*Address all correspondence to: gp2571@cumc.columbia.edu

1 CONICET-University of Buenos Aires, Institute of Immunology, Genetics and Metabolism (INIGEM), School of Pharmacy and Biochemistry, Clinical Hospital “José de San Martín”, Buenos Aires, Argentina

2 Department of General and Oral Biochemistry, School of Dentistry, University of Buenos Aires, Buenos Aires, Argentina

3 Department of Clinical Operative and Prosthesis II, School of Dentistry, University of Buenos Aires, Buenos Aires, Argentina

\section{References}

[1] Elsalanty ME, Genecov DG. Bone grafts in craniofacial surgery. Craniomaxillofacial Trauma and Reconstruction. 2009;2:125-134. DOI: 10.1055/s-0029-1215875

[2] Albee FH. The fundamental principles underlying the use of the bone graft in surgery. In: Saunders B, editor. Bone-graft surgery. Philadelphia and London: W. Company; 1915. pp. $17-51$

[3] Petite H, Viateau V, Bensaid W, Meunier A, De Pollak C, Bourguignon M, et al. Tissue engineered bone regeneration. Nature Biotechnology. 2000;18:959-963. DOI: 10.1038/79449

[4] Fuentes Fernández R, Bucchi C, Navarro P, Beltrán V, Borie E. Bone grafts utilized in dentistry: An analysis of patients' preferences. BMC Medical Ethics. 2015;16:71. DOI: 10.1186/s12910-015-0044-6 
[5] Wang WH, Yeung KWK. Bone grafts and biomaterials substitutes for bone defect repair: A review. Bioactive Materials. 2017;2:224-247

[6] Albrektsson T, Johansson C. Osteoinduction, osteoconduction and osseointegration. European Spine Journal. 2001;10:96-101. DOI: 10.1007/s005860100282

[7] Oryan A, Alidadi S, Moshiri A, Maffulli N. Bone regenerative medicine: Classic options, novel strategies, and future directions. Journal of Orthopaedic Surgery and Research. 2014;9:18. DOI: 10.1186/1749-799X-9-18

[8] Laurell L, Gottlow J. Guided tissue regeneration update. International Dental Journal. 1998;48:386-339

[9] Athanasiou VT, Papachristou DJ, Panagopoulos A, Saridis A, Scopa CD, Megas P. Histological comparison of autograft, allograft-DBM, xenograft, and synthetic grafts in a trabecular bone defect: An experimental study in rabbits. Medical Science Monitor. 2010;16:24-31

[10] Rogers GF, Greene AK. Autogenous bone graft: Basic science and clinical implications. The Journal of Craniofacial Surgery. 2012;23:323-327. DOI: 10.1097/SCS.0b013e318241dcba

[11] Olate S, de Oliveira GR, Jaimes M, Barbosa JRA. Osseous recovery in implant insertion and pre implant reconstructions. International Journal of Morphology. 2007;25:649-657

[12] Grover V, Kapoor A, Malhotra R, Sachdeva S. Bone allografts: A review of safety and efficacy. Indian Journal of Dental Research. 2011;22:496. DOI: 10.4103/0970-9290.87084

[13] Bostrom MP, Seigerman DA. The clinical use of allografts, demineralized bone matrices, synthetic bone graft substitutes and osteoinductive growth factors: A survey study. HSS Journal. 2005;1:9-18. DOI: 10.1007/s11420-005-0111-5

[14] Zimmermann G, Moghaddam A. Allograft bone matrix versus synthetic bone graft substitutes. Injury. 2011;(42):16-21. DOI: 10.1016/j.injury.2011.06.199

[15] Gomes KU, Carlini JL, Biron C, Rapoport A, Dedivitis RA. Use of allogeneic bone graft in maxillary reconstruction for installation of dental implants. Journal of Oral and Maxillofacial Surgery. 2008;66:2335-2338. DOI: 10.1016/j.joms.2008.06.006

[16] Muller MA, Frank A, Briel M, Valderrabano V, Vavken P, Entezari V, Mehrkens A. Substitutes of structural and non-structural autologous bone grafts in hind foot arthrodeses and osteotomies: A systematic review. BMC Musculoskeletal Disorders. 2012;14. DOI: 59. DOI: 10.1186/1471-2474-14-59

[17] Keating JF, McQueen MM. Substitutes for autologous bone graft in orthopaedic trauma. Journal of Bone and Joint Surgery. 2001;83:3-8

[18] Moshiri A, Oryan A. Role of tissue engineering in tendon reconstructive surgery and regenerative medicine: Current concepts, approaches and concerns. Hard Tissue. 2012;1:11

[19] Oryan A, Alidadi S, Moshiri A. Current concerns regarding healing of bone defects. Hard Tissue. 2013;2:13 
[20] Parikh SN. Bone graft substitutes: past, present, future. Journal of Postgraduate Medicine. 2002;48:142-148

[21] Anderson A, Mucalo MR, Johnson GS, Lorier MA. The processing and characterization of animal-derived bone to yield materials with biomedical applications. Part III: Material and mechanical properties of fresh and processed bovine cancellous bone. Journal of Materials Science: Materials in Medicine. 2000;11:743-749

[22] Johnson GS, Mucalo MR, Lorier MA. The processing and characterization of animalderived bone to yield materials with biomedical applications. Part I: Modifiable porous implants from bovine condyle cancellous bone and characterization of bone materials as a function of processing. Journal of Materials Science: Materials in Medicine. 2000;11:427-441

[23] Molly L, Vandromme H, Quirynen M, Schepers E, Adams JL, Van Steenberghe D. Bone formation following implantation of bone biomaterials into extractions sites. Journal of Periodontology. 2008;79:1108-1115. DOI: 10.1902/jop.2008.070476

[24] Wenz B, Oesch B, Horst M. Analysis of the risk of transmitting bovine spongiform encephalopathy though bone grafts derived from bovine bone. Biomaterials. 2001;22:1599-1606

[25] Accorsi-Mendoça T, Zambuzzi WF, Monteiro Bramante C, et al. Biological monitoring of xenomaterial for grafting: An evaluation in critical-sized calvarial defects. Journal of Materials Science: Materials in Medicine. 2011;22:997-1004. DOI: 10.1007/ s10856-011-4278-7

[26] Kao ST, Scott DD. A review of bone substitutes. Oral and Maxillofacial Surgery Clinics of North America. 2007;19:513-521. DOI: 10.1016/j.coms.2007.06.002

[27] Moore WR, Graves SE, Bain GI. Synthetic bone graft substitutes. ANZ Journal of Surgery. 2001;71:354-361

[28] Hench LL, Xynos ID, Polak JM. Bioactive glasses for in situ tissue regeneration. Journal of Biomaterials Science, Polymer. 2004;15:543-562

[29] Hench LL. The story of bioglass. Journal of Materials Science: Materials in Medicine. 2006;17:967-978. DOI: 29- 10.1007/s10856-006-0432-z

[30] Appleford MR, Oh S, Oh N, Ong L. In vivo study on hydroxyapatite scaffolds with trabecular architecture for bone repair. Journal of Biomedical Materials Research. 2009;89:1019-1027. DOI: 10.1002/jbm.a.32049

[31] Meimandi Parizi A, Oryan A, Shafiei-Sarverstani Z, et al. Effectiveness of synthetic hydroxyapatite versus Persian gulf coral in an animal model of long bone defect reconstruction. Journal of Orthopaedics and Traumatology. 2013;14:259-268. DOI: 10.1007/ s10195-013-0261-z

[32] Sugimori E, Shintani S, Ishikawa K, Ha-Makawa H. Effects of apatite foam combined with platelet-rich plasma on regeneration of bone defects. Dental Materials Journal. 2006; 25:591-596 
[33] Liu J, Kerns DG. Mechanisms of guided bone regeneration: A review. The Open Dentistry Journal. 2014;8:56-65

[34] Schropp L, Wenzel A, Kostopoulos L, Karring T. Cicatrización ósea y tejidos blandos tras la extracción de un solo diente: Un estudio clínico y radiográfico Estudio prospectivo de 12 meses. International Journal of Periodontics \& Restorative Dentistry. 2003;23:313-323

[35] He H, Yan W, Chen G, Lu Z. Acceleration of de novo bone formation with a novel bioabsorbable film: A histomorphometric study in vivo. Journal of Oral Pathology \& Medicine. 2008;37:378-382

[36] Zhang X, Cai Q, Liu H, Heng BC, Peng H, et al. Osteoconductive effectiveness of bone graft derived from antler cancellous bone: An experimental study in the rabbit mandible defect model. International Journal of Oral and Maxillofacial Surgery. 2012;41:1330-1337

[37] Andersson L, Ramzi A, Joseph B. Studies on dentin grafts to bone defects in rabbit tibia and mandible: Development of an experimental model. Dental Traumatology. 2009;25:78-83

[38] Xu S, Lin K, Wang Z, et al. Reconstruction of calcarial defect of rabbits using porous calcium silicate bioactive ceramics. Biomaterials. 2008;29:2588-2596

[39] Sohn JY, Park JC, Um YJ, et al. Spontaneous healing capacity of rabbit cranial defects of various sizes. Journal of Periodontal \& Implant Science. 2010;40:180-187

[40] Zeni SN, Di Gregorio S, Gomez Acotto C, Mautalen C. Olpadronate prevent the bone loss induced by cyclosporine in the rat. Calcified Tissue International. 2002;70:48-53

[41] Nemcovsky CE, Serfaty V. Alveolar ridge preservation following extraction of maxillary anterior teeth. Report on 23 consecutive cases. Journal of Periodontology. 1996;67:390-395

[42] Tatum OH. Maxillary and sinus implant reconstruction. Dental Clinics of North America. 1986;30:227-229

[43] Jarcho M. Calcium phosphate ceramics as a hard tissue prosthetics. Clinical Orthopaedics. $1981 ;(157): 259-278$

[44] Nudelman F, Pieterse K, George A, et al. The role of collagen in bone apatite formation in the presence of hydroxyapatite nucleation inhibitors. Nature Materials. 2010;9:1004-1009

[45] Kenkre JS, Bassett J. The bone remodeling cycle. Annals of Clinical Biochemistry. 2018; 55:308-327. DOI: $10.1177 / 0004563218759371$

[46] Figueiredo M, Henriques J, Martins G, Guerra F, Judas F, Figueiredo HJ. Physicochemical characterization of biomaterials commonly used in dentistry as bone substitutesComparison with human bone. Journal of Biomedical Materials Research. Part B, Applied Biomaterials. 2010;92:409-419. DOI: 10.1002/JBM.b.31529

[47] Chackartchi T, Iezzi G, Goldstein M, et al. Sinus floor augmentation using large (1-2 mm) or small $(0.25-1 \mathrm{~mm})$ bovine bone mineral particles: A prospective, intra-individual controlled clinical, micro-computerized tomography and histomorphometric study. Clinical Oral Implants Research. 2011;22:473-480 
[48] Chiapasco M, Zaniboni M. Methods to treat the edentulous posterior maxilla: Implants with sinus grafting. Journal of Oral and Maxillofacial Surgery. 2009;67:867-871. DOI: 10.1016/j.joms.2008.11.023

[49] Block MS, Kent JN. Sinus augmentation for dental implants: The use of autogenous bone. Journal of Oral and Maxillofacial Surgery. 1997;55:1281-1286

[50] Schulze-Späte U, Dietrich T, Wu C, Wang K, Hasturk H, Dibart S. Systemic vitamin D supplementation and local bone formation after maxillary sinus augmentation - A randomized, double-blind, placebo-controlled clinical investigation. Clinical Oral Implants Research. 2016;27:701-706

[51] Shirmohammadi A, Roshangar L, Taghi Chitsazi M, Pourabbas R, Faramarzie M, Rahmanpour N. Comparative study on the efficacy of anorganic bovine bone (Bio-Oss) and nanocrystalline hydroxyapatite (Ostim) in maxillary sinus floor augmentation. International Scholarly Research Notices. 2014. Article ID: 967091

[52] Lyndon F, Cooper LF. The current and future treatment of edentulism. Journal of Prosthodontics. 2011;18:116-122

[53] Wallace SS, Froum SJ, Cho SC, Elian N, Monteiro D, Kim BS, Tarnow DP. Sinus augmentation utilizing anorganic bovine bone (Bio-Oss) with absorvable and nonabsorbable membranes placed over the lateral window: Histomorphometric and clinical analyses. The International Journal of Periodontics \& Restorative Dentistry. 2005;25:551-559 
